\title{
A rule-based presentation order facilitates category learning
}

\author{
FABIEN Mathy \\ Université de Franche-Comté, Besançon, France \\ AND \\ JACOB FELDMAN \\ Rutgers University, New Brunswick, New Jersey
}

\begin{abstract}
We investigated the mechanisms by which concepts are learned from examples by manipulating the presentation order in which the examples were presented to subjects. We introduce the idea of a rule-based presentation order, which is a sequence that respects the internal organization of the examples within a category. We find that such an order substantially facilitates learning, as compared with previously known beneficial orders, such as a similarity-based order. We discuss this result in light of the central distinction between rule-based and similaritybased learning models.
\end{abstract}

A number of studies have investigated whether category learning is influenced by the order in which examples are presented. Elio and Anderson (1981) found that categories are learned faster when training is blocked into groups of mutually similar examples (see also Elio \& Anderson, 1984). More recently, Medin and Bettger (1994) demonstrated a strong learning advantage when training objects were presented in an order that tended to maximize similarity between successive examples. Other studies, such as those of Clapper and Bower (1994) and Goldstone (1996), have focused on the effect of alternation of contrasting categories. Presentation order effects are especially interesting in the light of categorization models that emphasize incremental learning from trial to trial. For example, Sakamoto, Jones, and Love (2008) showed that order can affect the incremental update of both category means and variances (see also Love, Medin, \& Gureckis, 2004). Incremental-learning models are naturally susceptible to order effects, whereas other models may be less so, so the manipulation of presentation order is a potentially useful tool for studying the mechanisms of learning.

However, previous studies of presentation order were limited in that they used orders based on simple similarity - for example, maximizing or minimizing the similarity between adjacent training examples. Here, we explore a type of presentation order that depends in a more structured way on the nature of the category to be learned. We introduce the notion of a rule-based presentation order, which is one that derives from the internal structure of the training examples. In our rule-based order, objects that are within a rule - that is, that obey the same structured subclass within the category-are presented adjacently in the presentation sequence. Training then moves on to another subclass, and so forth until all the objects have been presented. (Negative instances are randomly interspersed among the positives; only the order of the positives is manipulated.) Below, we will compare subjects' performance with such an order with the similarity-based order found to be advantageous in earlier studies. For comparison, we will also include a dissimilarity-based order, previously found to be disadvantageous. We hypothesize that the rulebased order will facilitate learning, particularly in highly structured concepts (i.e., those containing more clusters), by aiding the subject in mentally organizing what would otherwise appear heterogeneous or chaotic.

\section{METHOD}

\begin{abstract}
Subjects
The subjects were 96 Rutgers University students who received course credit in exchange for their participation.

\section{Procedure}

Tasks were computer-driven. The subjects learned to sort stimulus objects using two keys, with successful learning encouraged by means of a progress bar. Stimulus objects were presented one at a time in the upper part of the computer screen. After each response, feedback indicating a correct or incorrect classification was provided at the bottom of the screen for $2 \mathrm{sec}$. The subjects learned a simple concept in two dimensions as a short warm-up session. Then each subject was asked to learn the two chosen concepts (details below). The order of the two concepts was counterbalanced between subjects. For each subject, a single presentation order was randomly chosen and applied to the two concepts. The three different presentation orders will be described in more detail below.
\end{abstract}



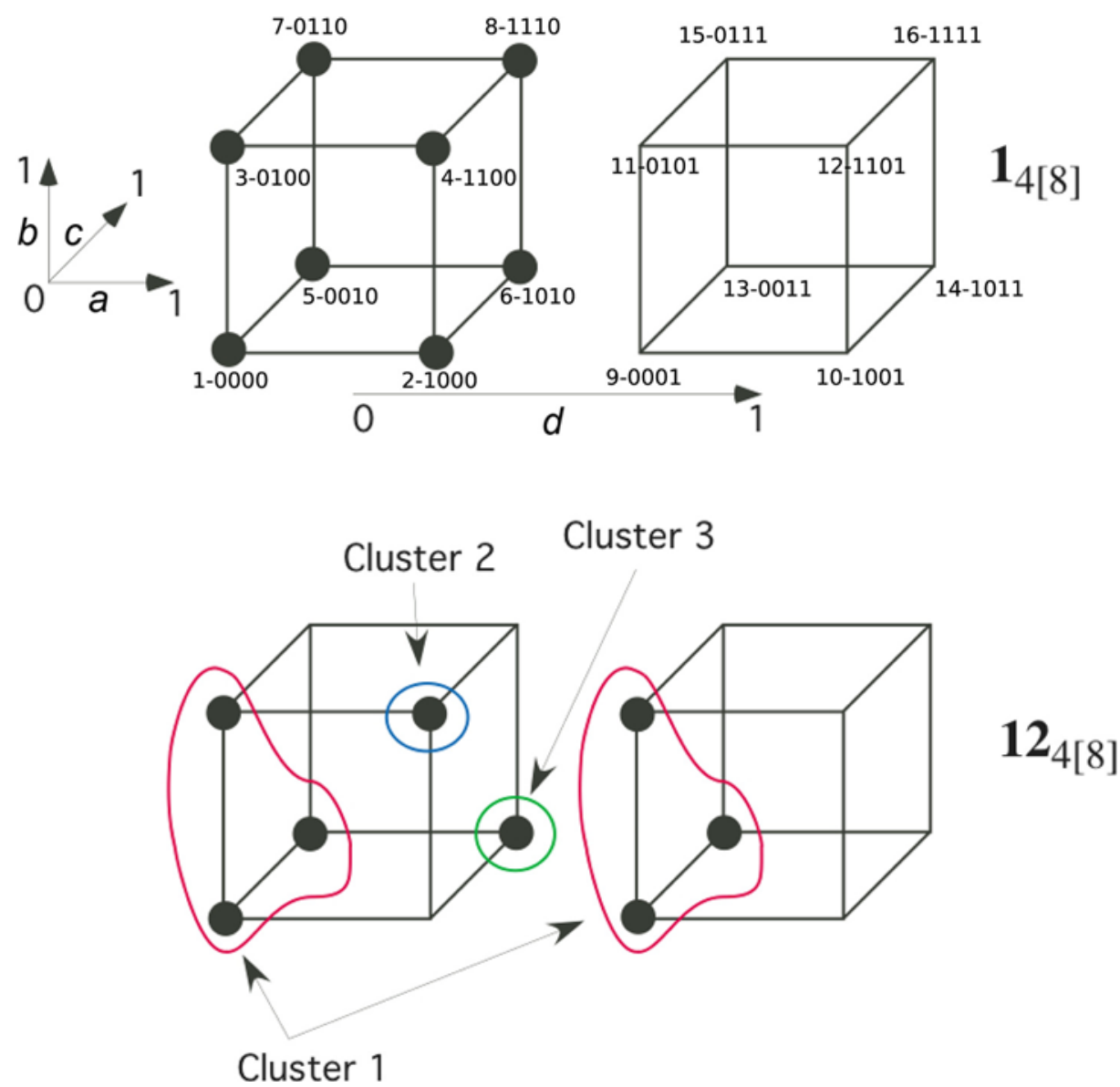

Figure 1. Concepts $\mathbf{1}_{4[8]}$ and $12_{4 \mid 8]}$ of the $4[8]$ family (see Feldman, 2000, for a further explanation of the concept of family taxonomy). Positive examples are indicated by black circles; negative examples are represented by empty vertices. There is one cluster in Concept $\mathbf{1}_{4|8|}$ and three clusters in Concept $12_{4|8|}$. The stimulus coding order is $a b c d$. The code 0000 stands for $a^{\prime} b^{\prime} c^{\prime} d^{\prime} ; 1111$ stands for $a b c d$. The number preceding the code is a simpler identification number.

Each correct response scored them 1 point in a progress bar. The point was represented by an empty box that was filled in when the subjects gave a correct response. To regulate the learning process, each response had to be given in less than $8 \mathrm{sec}$ (making a maximum of $10 \mathrm{sec}$ between two stimuli when the subjects got a "Too late" message feedback, itself lasting $2 \mathrm{sec}$ ). If the response was given too late, the subjects would lose 3 points on the progress bar. The number of points in the progress bar dedicated to learning was $4 \times 2^{D}(D=$ number of dimensions, four in our study). This criterion was identical to the one used by Shepard, Hovland, and Jenkins (1961) in their first experiment. Consequently, the subjects had to correctly classify stimuli on four consecutive blocks of $2^{D}$ stimuli. ${ }^{1}$

\section{Choice of Concepts Studied}

Each of the subjects was given two concepts to learn, each defined over four dimensions (shown schematically in Figure 1). We used four-dimensional concepts so that the number of objects to be classified $\left(2^{4}=16\right)$ would be large enough to bring out any effects of our manipulations of presentation order, but small enough to be manageable in a single experimental session. We chose to focus on Types $1_{4[8]}$ and $12_{4[8]}$ (Figure 1) of the typology of Feldman (2003; an extension of those in Shepard et al., 1961, and Feldman, 2000). In this notation, the [8] extension means that there are eight positive examples in the concept, 4 means that the concept is based on four dimensions, and the 1 and 12 are arbitrary labels that identify these concepts from among the 72 other concepts available. ${ }^{2}$

The two concepts are defined up to isomorphism, respectively, by the formulae

$$
1_{4[8]} \cong d^{\prime}
$$

and

$$
12_{4[8]} \cong a^{\prime}(b c)^{\prime}+a d^{\prime}\left(b c^{\prime}+b^{\prime} c\right) .
$$

Here, we use a standard notation in which $a^{\prime}$ refers to negation of feature or clause $a, a b$ refers to the conjunction of $a$ and $b$, and $a+b$ refers to their disjunction (equivalent to but more concise and readable than the $\neg, \wedge, \vee$ notation often used). In Figure 1, the concepts are shown in an arbitrary rotation and permutation of features; as will be explained below, this mapping was randomized in the experiment. The concepts are also encoded and represented linearly in Table 1.

The symbol $\cong$ in the equations above indicates congruence or structural isomorphism up to this arbitrary mapping. For example the concepts $a, a^{\prime}, b, \ldots$, or $d^{\prime}$ are all congruent because they are equivalent after relabeling of the features; in each case, exactly one value of one feature defines the concept. Concept $1_{4[8]}$, because it can be expressed by a single literal, has a complexity of 1 and is thus the simplest concept in the 4[8] family, equivalent to affirmation (assertion of the presence of a single feature) in the classical literature (the four-dimensional analogue of Type I from Shepard et al., 1961). 
Table 1

Encoded Study Items Presented in Figure 1 and Presentation Order Samples in Concept $\mathbf{1}_{4[8]}$

\begin{tabular}{|c|c|c|c|c|c|c|c|c|c|}
\hline \multicolumn{2}{|c|}{$1_{4[8]}$} & \multicolumn{2}{|c|}{$12_{4[8]}$} & \multicolumn{6}{|c|}{ Presentation Order Samples in $1_{4[8]}$} \\
\hline \# & Cat 1 & \# & Cat 1 & & BO & & BO & & $\mathrm{BO}$ \\
\hline 1 & 0000 & 1 & 0000 & 1 & 0000 & - & Cat 0 & 3 & 0100 \\
\hline 2 & 1000 & 3 & 0100 & 2 & 1000 & 8 & 1110 & 5 & 0010 \\
\hline 3 & 0100 & 4 & 1100 & - & Cat 0 & - & Cat 0 & 7 & 0110 \\
\hline 4 & 1100 & 5 & 0010 & 4 & 1100 & 1 & 0000 & - & Cat 0 \\
\hline 5 & 0010 & 6 & 1010 & - & Cat 0 & - & Cat 0 & - & Cat 0 \\
\hline 6 & 1010 & 9 & 0001 & 3 & 0100 & 4 & 1100 & 8 & 1110 \\
\hline 7 & 0110 & 11 & 0101 & 7 & 0110 & 5 & 0010 & 4 & 1100 \\
\hline 8 & 1110 & 13 & 0011 & 8 & 1110 & - & Cat 0 & - & Cat 0 \\
\hline & & & & - & Cat 0 & 3 & 0100 & 1 & 0000 \\
\hline \# & Cat 0 & \# & Cat 0 & - & Cat 0 & 6 & 1010 & - & Cat 0 \\
\hline 9 & 0001 & 2 & 1000 & 6 & 1010 & - & Cat 0 & - & Cat 0 \\
\hline 10 & 1001 & 7 & 0110 & 5 & 0010 & - & Cat 0 & 2 & 1000 \\
\hline 11 & 0101 & 8 & 1110 & - & Cat 0 & 7 & 0110 & 6 & 1010 \\
\hline 12 & 1101 & 10 & 1001 & & Cat 0 & - & Cat 0 & - & Cat 0 \\
\hline 13 & 0011 & 12 & 1101 & - & Cat 0 & 2 & 1000 & - & Cat 0 \\
\hline 14 & 1011 & 14 & 1011 & - & Cat 0 & - & Cat 0 & - & Cat 0 \\
\hline 15 & 0111 & 15 & 0111 & & & & & & \\
\hline 16 & 1111 & 16 & 1111 & & & & & & \\
\hline
\end{tabular}

Note-In the "Presentation Order Samples in $1_{4[8]}$ " columns, Cat 0 cells can be replaced by any negative examples of the Cat 0 category, because negative examples were drawn in random order. SBO, similarity-based order; DBO, dissimilarity-based order; RBO, rule-based order; Stimulus \# is also indicated in Figure 1.

The second concept, $12_{4[8]}$, has a complexity of 9 literals and is thus of moderate complexity, relative to others in the 4[8] family. (Complexity of concepts in this family ranges from 1 to 22 literals.) We chose this concept for several reasons. First, we wanted a moderately complex concept, so that the entire learning procedure could be completed by most subjects in about an hour. Among concepts of moderate complexity, we chose $12_{4[8]}$ because its positive examples can be grouped fairly naturally into subcategories or clusters (labeled Clusters 1, 2, and 3 in Figure 1), allowing us to investigate the interaction between presentation order and such internal substructure. As can be seen in the figure, Cluster 1 comprises six of the concept's eight members, corresponding to the first disjunctive clause $\left[a^{\prime}(b c)^{\prime}\right]$ in the concept's compressed formula. Thus, these six objects collectively receive an extremely compact expressiona clause of only 3 literals (which can be translated into a verbal expression, such as "all $a^{\prime}$ except $b c$ "). By contrast, Clusters 2 and 3 consist of only one object each, each requiring 4 literals to specify just by themselves $\left(a b c^{\prime} d^{\prime}\right.$ and $a b^{\prime} c d^{\prime}$, respectively, corresponding to the expansion of the second clause in the formula). Thus, Cluster 1 plays the role of a salient rule, whereas Clusters 2 and 3 play the role of exceptions.

Our presentation order was based on the presupposition that the subjects would cluster the members of Concept $12_{4[8]}$ in the manner given above. This a reasonable assumption - in part, because this clustering corresponds to a highly compressed Boolean form, consistent with the minimization of Boolean complexity (Feldman, 2000). Naturally, though, this concept (like any other) admits other interpretations or subclusterings, and we have no way of confirming that our subjects mentally organized it in the way that we expected (other than the fact that the presentation order based on this decomposition did, in fact, benefit learning, as will be seen below). However, any alternative subclustering that the subjects might have drawn would simply have added noise to our analysis, working against our hypothesis, so our assumption was conservative.

\section{Stimuli}

Stimulus objects varied along four binary, separable dimensions (shape, color, size, and filling texture). For each concept (desig- nation of eight positive and eight negative objects), assignment of abstract conceptual structures to physical features was randomized; that is, the abstract features $a, b, c, d$ were randomly permuted before being realized as physical features. Also, for each concept, the choice of two values for each feature was chosen randomly from a sometimes longer list (shape $=$ triangle, square, or circle; color $=$ blue, pink, red, or green; filling $=$ hatched or grilled; size $=$ small or big). Each of the 16 combinations of values formed a single unified object (e.g., a small hatched red square or a big grilled blue circle) to avoid numerical or spatial biases when displaying stimuli.

\section{Ordering of Stimuli}

The main manipulation in the experiment was the choice of presentation order of objects within each concept. Presentation order was a between-subjects manipulation. Again, one presentation order was randomly chosen for a given subject and then applied to the two concepts. We used three orders: a rule-based order, a similarity order, and a dissimilarity order. As was discussed above, Concept $12_{4[8]}$ consists of a single relatively coherent cluster of six objects and two "clusters" of one object each. By contrast, Concept $1_{4[8]}$ consists of a single homogeneous cluster.

In each of the three order types, objects were drawn without replacement in each block of 16; that is, each block of 16 consisted of a complete permutation of all the objects. The negative examples were randomly interspersed among the positives (in random order) in order to avoid long uninterrupted sequences of positives or negatives, even though this presumably made it more difficult for the subjects to benefit from the presentation orders. Again, only the order of the positive examples was manipulated, taking one of the orders detailed below, whereas the order of the negatives was always random (and thus different from block to block and subject to subject)

In the rule-based order, objects were drawn randomly from within the largest cluster (in $1_{4[8]}$, the entire concept; in $12_{4[8]}$, Cluster 1 ) until all eight $\left(1_{4[8]}\right)$ or six $\left(12_{4[8]}\right)$ had been presented. In Concept $1_{4[8]}$, this would exhaust the entire concept, whereas in Concept $12_{4[8]}$, this would be followed by the objects in Cluster 2 and Cluster 3 (in random order). Thus, in the rule-based order, all members obeying a common rule were presented together, in random order but separated from exceptional members. ${ }^{3}$

In the similarity order, the first object was chosen at random, subsequent objects were chosen randomly from those maximally similar to the previous object, and so forth, until the concept was exhausted. ${ }^{4}$ Ties were resolved randomly. Dissimilarity between two stimuli $i$ and $j$ is given by the Minkowski metric

$$
d_{i j}=\left[\sum_{a=1}^{n}\left|x_{i a}-x_{j a}\right|^{r}\right]^{1 / r},
$$

where $x_{i a}$ is the value of stimulus $i$ along dimension $a$. We used a city-block metric appropriate for separable dimensions used in this study $(r=1)$. In general, this similarity ordering does not respect the cluster boundaries in force in the rule-based order, since similarity steps routinely cross in and out of clusters in $12_{4[8]}$. The similarity order also differs from the rule-based order in that (aside from ties) the steps are not random.

In the dissimilarity order, objects were drawn exactly as in the similarity order, except with similarities minimized instead of maximized. That is, each object would be followed by another object as distant as possible from it in the space.

In all three orders, each new block of 16 was newly randomized (the positive instances were randomly drawn but constrained to obey the desired order, and the negative instances were randomly interspersed), so the subjects rarely saw consistent specific sequences of objects between blocks.

\section{Comparison With Procedures Used in Other Studies}

In Elio and Anderson's (1981) similarity-based order, the presentation order increased interitem similarity, relative to a random se- 
quence, but did not maximize it. Our similarity-based order is based on the Minkowski metric, with no distinction of any sort between examples except their similarity. Items are simply chosen so that they maximize (or in the dissimilarity order, minimize) the similarity to the previous item. In this sense, our similarity-based order is more extreme than Elio and Anderson's (1981) but also more varied than the one used by Medin and Bettger (1994), who used a single fixed similarity-based order and dissimilarity-based order in each of their experiments. Our procedure produces a locally maximal interitem similarity in the similarity-based order and a minimal interitem similarity in the dissimilarity-based order. (Numbers will be given in the Results section.) In comparison, the interitem similarity in the rule-based order is moderate. Some presentation order samples are given in Table 1. Finally, note also that because subjects are not aware of where the blocks begin, they might be more sensitive to the isolation of the stimuli belonging to different clusters in the rulebased ordering than to the strict position of the clusters in the blocks (related to the von Restorff isolation effect, transposed to categorization in Sakamoto \& Love, 2004).

\section{RESULTS}

The average interitem similarity within the similaritybased order, the dissimilarity-based order, and the rulebased order was, respectively, 2.9, 1.5, and 2.3 for Concept $1_{4[8]}$ and $2.7,1.3$, and 2.1 for Concept $12_{4[8]} .^{5}$ These empirical measures correspond to what would be expected in principle. For example, in Concept $1_{4[8]}$, the similarity between two positive stimuli is most of the time equal to 3 in the similarity-based order (once in a while, it can be equal to 2 or 1 at the end of certain blocks, when the path does not allow any other choice), except between the last stimulus of a given block and the first stimulus of the next block (the first stimulus of a block being drawn randomly). The average interitem similarity between the last stimulus of the $n$th block and the first of the $(n+1)$ st block is 2.5 (i.e., the average of $4,3,3,3,2,2,2,1$ ). Therefore, the theoretical average interitem similarity in Con- cept $1_{4[8]}$ is $(7 \times 3+1 \times 2.5) / 8 \approx 2.9$, agreeing with the empirical measure. These measures will be used for comparison purposes in the following analysis.

We will first consider the influence of presentation order on learning and then will provide more detailed analyses of the progression of learning over time and, finally, of classification response times.

\section{Influence of Presentation Order on Learning}

Among the 96 subjects ( 32 by presentation order), only 69 subjects could finish the experiment within the time slot allocated to the experiment. The analysis of results takes into account only these 69 subjects ( 25 for the rulebased procedure, 21 for the dissimilarity order, and 23 for the similarity order). The loss of subjects apparently tracked the difficulties associated with the presentation orders presented below, although the chi-square test of independence between presentation order and loss was not significant $\left[\chi^{2}(2)=1.2\right.$, n.s. $]{ }^{6}$

Figure 2 shows the number of blocks that were required for the subjects to reach the learning criterion in the three conditions (rule based, similarity based, and dissimilarity based) for both concepts $\left(1_{4[8]}\right.$ and $\left.12_{4[8]}\right)$ : respectively, 5.2, 6.1, and 6.9 blocks for Concept $1_{4[8]}$ and 22.1, 28.1, and 42.6 blocks for Concept $12_{4[8]}$. The results indicate that there was an effect of the presentation order on learning: The number of blocks required to reach the learning criterion depended on the presentation order that was chosen $\left[F(2,66)=15.3, p<.001, \eta_{\mathrm{p}}^{2}=.32\right]$. Learning was fastest in the rule-based order $(M=13.7$ blocks until criterion, $S D=10.4)$, second fastest in the similarity-based condition $(M=17.1$ blocks, $S D=12.6)$, and slowest in the dissimilarity-based condition $(M=24.7$ blocks, $S D=$ 22.5). The superiority of the similarity over dissimilarity order replicates earlier findings. But the main result, that the rule-based ordering is superior to either, is novel.

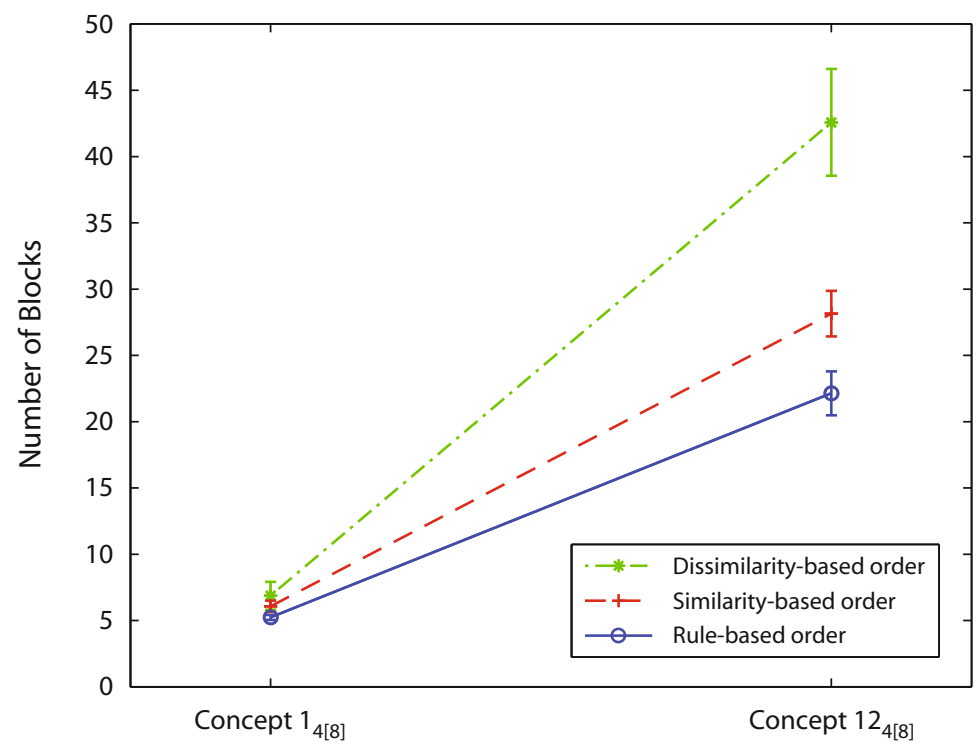

Figure 2. Number of blocks taken to reach the learning criterion of $100 \%$ correct classification for two consecutive blocks. Error bars show $\pm 1 S E$. 


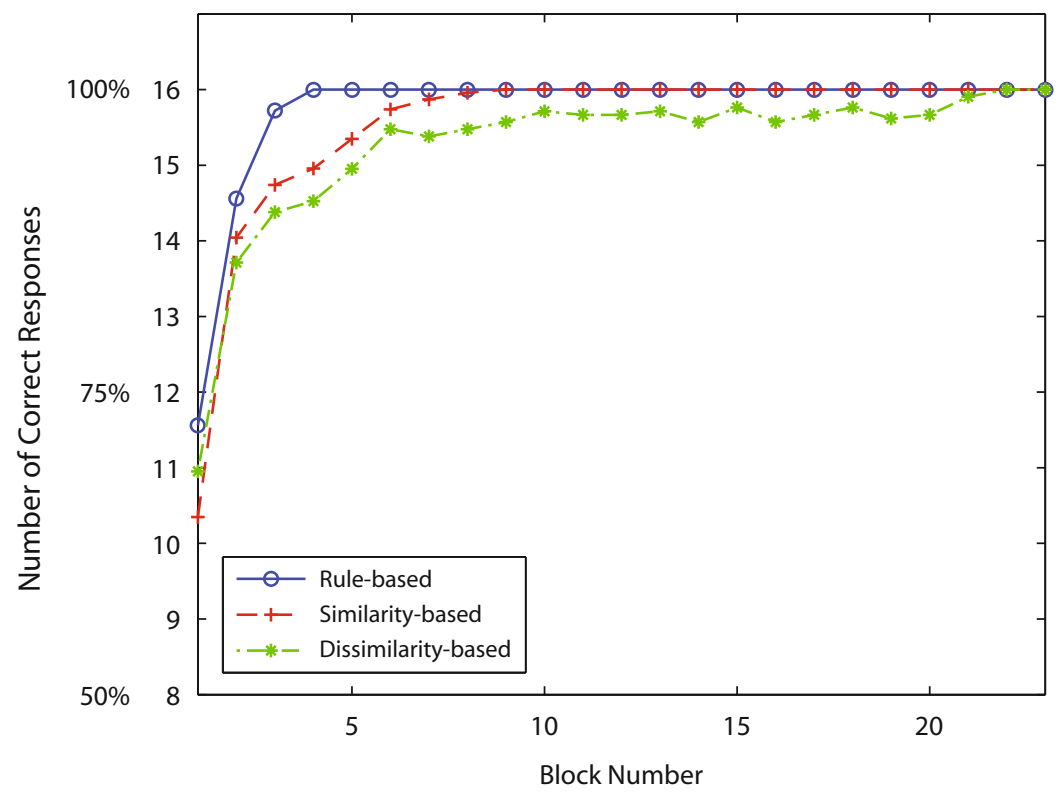

Figure 3. Performance as a function of block number for the three presentation orders, Concept $1_{4|8|}$. To compare any pair of learning curves, we fitted the data sets to a common nonlinear model $y=b_{0}+b_{1} / x$, which fit all three data sets well $\left(R^{2}>.89\right.$ in all cases). We tested the null hypothesis of equal slopes between pairs of regression curves by comparing the mean squared error when the two data sets were pooled with when they were fit separately (the technique is similar to verifying that there is no interaction between the covariate and treatments before running an ANCOVA). The results showed that the three learning curves are statistically distinct.

As can be seen in Figure 2, Concept $12_{4[8]}$ was learned much more slowly overall $[F(1,66)=320, p<.001$, $\left.\eta_{\mathrm{p}}^{2}=.83\right]$. The effect of presentation order was far larger in magnitude in this complex concept, which is reflected in the interaction between concepts and presentation orders $\left[F(2,66)=15.8, p<.001, \eta_{\mathrm{p}}^{2}=.32\right]$. When simple effects of presentation orders were analyzed for each concept, the effect of presentation order was significant only in Concept $12_{4[8]}\left[F(2,66)=16.34, p<.001, \eta_{\mathrm{p}}^{2}=.33\right]$. The between-subjects $t$ tests indicated that only the three paired comparisons between presentation orders for Concept $12_{4[8]}$ were significant. However, a subsequent analysis of learning curves will reveal that presentation order also influences learning of Concept $1_{4[8]}$.

\section{Detailed Analysis of the Progression of Learning}

We next will turn to the question of how learning progresses over time in the three presentation orders. Figure 3 (Concept $1_{4[8]}$ ) and Figure 4 (Concept $12_{4[8]}$ ) show both the percentage and the number of correct responses for each block as a function of block number over the course of the experiment. Note that the absolute number is sometimes more revealing than the percentage; for example, 14 correct responses $(87.5 \%)$ can be immediately understood to mean "all but 2 in the concept."

Concept $\mathbf{1}_{4[8]}$. As can be seen in Figure 3, learning of the simpler Concept $1_{4[8]}$ was slightly more efficient in the rulebased than in the similarity order. The fit comparison procedure, described in the notes of Figure 3, indicated that the two curves differ significantly in fitted form $\left[F_{\mathrm{RS}}(42,44)=\right.$ 13.17, $p<.001]$. Thus, even in Concept $1_{4[8]}$, although the difference is relatively subtle, the rule-based order produces significantly more rapid learning than does the similarity order. In contrast, the dissimilarity order seems to have induced ineffective learning, as compared with the similaritybased order $\left[F_{\mathrm{DS}}(42,44)=19.25, p<.001\right]$ and the rulebased order $\left[F_{\mathrm{DR}}(42,44)=18.46, p<.01\right]$.

Concept $\mathbf{1 2}_{4[8]}$. Using a similar fit comparison procedure, the same ranking of effectiveness of the three presentation orders (rule based $>$ similarity $>$ dissimilarity) was visible in the learning curves in Concept $12_{4[8]}$ [again, much larger in magnitude than in the simpler concept; we obtained $F(144,147)>40, p<.001$, for the three paired comparisons of the learning curves].

\section{DISCUSSION}

Like several previous studies, this study demonstrated that the sequence in which examples are encountered can profoundly influence the success of learning. Our results showed that a rule-based order yields learning superior to that of the similarity order previously found most advantageous (Medin \& Bettger, 1994). Although our interest was primarily theoretical, this result has obvious implications for the presentation of material in educational settings (Avrahami, 1987).

Our results demonstrated that the benefit of the rulebased order does not derive entirely from the interitem 


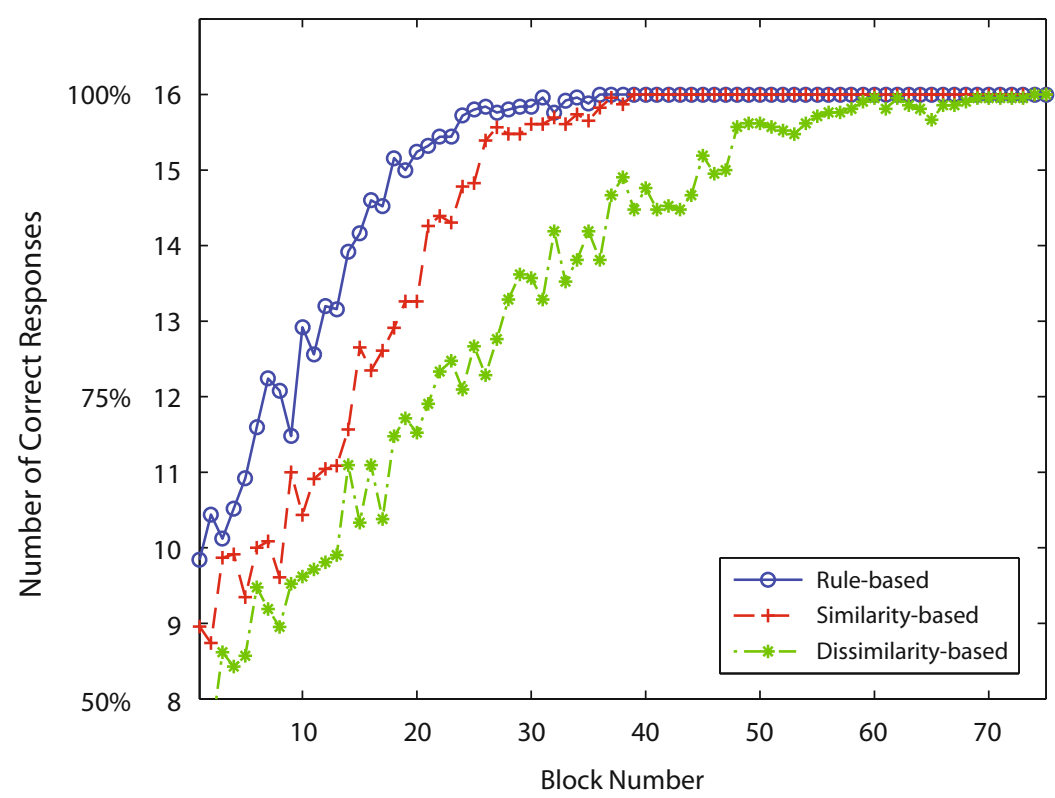

Figure 4. Performance over time (blocks) for the three presentation orders, Concept $12_{4|8|}$. The three learning curves are statistically distinct. The fit comparison procedure is described in the caption of Figure 3. This time, we fitted the data sets to a quadratic model $y=b_{0}+b_{1} x+b_{2} x^{2}$, which fit all three data sets well $\left(R^{2}>.92\right.$ in all cases).

similarity it entails. Interitem similarity was maximal in the similarity-based order and only intermediate in the rule-based order, but the rule-based order elicited the best performance. We conclude that the nature of the rule-based order (i.e., randomness and clustering) provides an independent learning benefit above and beyond that provided by interitem similarity. Nevertheless, in order to affirm that the nature of rule-based orders was critical in the performance we observed, subsequent experiments will be needed to show more precisely that, with an equivalent interitem similarity, subjects would perform worse in a non-rule-based order than in a rulebased order. Note that a reduction in between-blocks order variability (as a consequence of presenting the six positive items of the largest cluster before the two remaining exceptions on every block) in Concept $12_{4[8]}$ for the rule-based order is very unlikely to be a source of facilitation for subjects. There are $6 !=720$ possible orders for the six examples of the largest cluster. The subjects could not rely on a reduced number of possible orders to come up with a strategy.

This superiority of the rule-based order over the similarity order might be attributed to the illusory structure that the similarity order might tend to induce in the minds of learners. The similarity condition entails a relatively orderly trajectory through the space that is, in fact, not genuinely informative about the category and, thus, might temporarily mislead the learner about the structure to be learned. By contrast, the rule-based order, by definition, randomizes that which is not informative (steps within a cluster) while segregating the clusters, yielding superior learning.
These results suggest that human learning does involve a process of rule-based abstraction, consistent with many recent hybrid models (Anderson \& Betz, 2001; Erickson \& Kruschke, 1998; Goodman, Tenenbaum, Feldman, \& Griffiths, 2008; Nosofsky, Palmeri, \& McKinley, 1994; Rosseel, 2002; Smith \& Sloman, 1994) or clustering models such as SUSTAIN (Love et al., 2004), which overtly involve a rule-like component. (SUSTAIN also involves an incremental functioning, which can be responsible for overspecific solutions when the items are presented in an unfavorable order.) It also might be consistent with pure exemplar-storage schemes (Estes, 1994; Kruschke, 1992; Medin \& Schaffer, 1978; Nosofsky, Gluck, Palmeri, McKinley, \& Glauthier, 1994), since these are also sensitive to the homogeneity of categories (Hintzman, 1986), although the connection would be less direct. In any case, exemplar models are generally batch models (i.e., in batch algorithms, categorization probabilities are not computed trial by trial but, for the whole set of examples, block after block), meaning that they consider the set of examples as an unordered group and, hence, cannot model our results without some sort of extension. One exception is ALCOVE (Kruschke, 1992), because it includes trial-by-trial updating. AMBRY (Kruschke, 1996), derived from ALCOVE, might also be adequate for fitting our results, because it allows changes of category-to-response association weights and exemplar-to-category association weights after every trial in order to model rapid shifting in categorization. We feel that the superiority of rule-based presentation orders is a key test for models of categorization learning - a finding that any competitive model needs to be able to account for in principle. The comparative 
benefits of different orders may be helpful in deciding among competing rule-based models, which differ in the nature of the rules extracted (Bradmetz \& Mathy, 2008; Feldman, 2000; Lafond, Lacouture, \& Mineau, 2007; Vigo, 2006; see also Love et al., 2004; Nosofsky, Palmeri, \& McKinley, 1994). That is, alternative rule-based orders could be devised to match the abstraction or compression techniques entailed by the various theories. Following our argument, the most effective presentation order for subjects would be that which accords with the subjects' own internal hypotheses or representations.

Of course, there is no guarantee that any given presentation order induces in subjects anything like the mechanisms involved in its construction. A rule-based order does not necessarily induce the formation of rules, nor does a similarity-based order necessarily induce the computation of similarity; nor, for that matter, does a random presentation necessarily induce anything like random guessing or rote memorization. Both the learning mechanisms hypothesized in rule-based models and those hypothesized in exemplar models capitalize on the structure present in the observations, although in different ways.

The differences among presentation orders depend in an interesting way on the nature of the concepts learned. Most obviously, the rule-based presentation order provides a substantial benefit only if the category is highly structured - that is, when it contains salient subcategories around which the presentation order can be organized. For concepts like $1_{4[8]}$ that lack internal subdivisions, a rule-based presentation is, in effect, a random presentation. But a similarity order might induce a sequence of temporary overspecific hypotheses (blind alleys) based on accidentally contiguous examples, which would impede learning.

We noted a particular negative effect of the dissimilaritybased order, in which subsequent positive examples were chosen so as to be as distant as possible from each other. Research on the effect of the relative magnitude information may account for this result (Stewart, Brown, \& Chater, 2002). Stewart et al. showed that categorization of a stimulus on trial $n$ is influenced by the stimulus and response on trial $n-1$, when information about presentation orders is not discarded. Their memory and contrast model predicts that subjects tend to respond with a different category when the difference between two consecutive examples is large.

\section{CONCLUSION}

We believe that the present investigation is an important step in understanding the effect of presentation order, but several new conditions need to be explored to gain additional insight. A first extension would be to separate the training phase (in which the presentation order is manipulated) from the categorization phase. This would allow the positive examples to be presented alone, rather than being interspersed with negative examples, which muddies the desired order.

We also plan to model our results, both with existing incremental models and with extensions of existing models.
Exemplar models might be extended to take the temporal dimension into account by including sequential order as a feature in similarity comparisons. The similarity between two stimuli would then be influenced by their relative serial position, inducing a neighborhood structure in terms of both features and time. The model could then capitalize on the temporal dimension to assess local distinctiveness, in the same way serial position effects are modeled as discrimination problems in serial or free recall (Brown, Neath, \& Chater, 2007). Another solution proposed by Stewart et al. (2002) is to adapt an exemplar model to predict sequence effects by weighting the stimulus on the previous trial more heavily than others in the summed similarity calculations, on the grounds that recent stimuli are more available in memory, or simply that they ought to be weighed more heavily in decisions.

\section{AUTHOR NOTE}

This research was supported, in part, by a postdoctoral research grant from the Fyssen Foundation to F.M. and by NSF Grant 0339062 to J.F. We are grateful to Jennifer Biddick, Jonathan Geis, and Jing Tien for assistance in data collection, and to Cordelia Aitkin, Erica Briscoe, and David Fass for helpful discussions. Correspondence concerning this article should be addressed to F. Mathy, 30-32 rue Mégevand, 25030 Besançon Cedex, France (e-mail: fabien.mathy@univ-fcomte.fr).

\section{REFERENCES}

Anderson, J. R., \& Betz, J. (2001). A hybrid model of categorization. Psychonomic Bulletin \& Review, 8, 629-647.

Avrahami, J. (1987). Teaching by examples: Implications for the process of category acquisition. Quarterly Journal of Experimental Psychology, 50A, 586-606.

Bradmetz, J., \& Mathy, F. (2008). Response times seen as decompression times in Boolean concept use. Psychological Research, 72, 211-234.

Brown, G. D. A., Neath, I., \& Chater, N. (2007). A temporal ratio model of memory. Psychological Review, 114, 539-576.

Clapper, J. P., \& Bower, G. H. (1994). Category invention in unsupervised learning. Journal of Experimental Psychology: Learning, Memory, \& Cognition, 20, 443-460.

Elio, R., \& ANDERSON, J. R. (1981). The effects of category generalizations and instance similarity on schema abstraction. Journal of Experimental Psychology: Human Learning \& Memory, 7, 397-417.

Elio, R., \& ANDERSON, J. R. (1984). The effects of information order and learning mode on schema abstraction. Memory \& Cognition, 12 , 20-30.

ERICKSON, M. A., \& KruschKe, J. K. (1998). Rules and exemplars in category learning. Journal of Experimental Psychology: General, 127, 107-140.

Estes, W. K. (1994). Classification and cognition. New York: Oxford University Press.

Feldman, J. (2000). Minimization of Boolean complexity in human concept learning. Nature, 407, 630-633.

Feldman, J. (2003). A catalog of Boolean concepts. Journal of Mathematical Psychology, 47, 75-89.

Goldstone, R. L. (1996). Isolated and interrelated concepts. Memory \& Cognition, 24, 608-628.

Goodman, N. D., Tenenbaum, J. B., Feldman, J., \& Griffiths, T. L. (2008). A rational analysis of rule-based concept learning. Cognitive Science, 32, 108-154.

Hintzman, D. L. (1986). "Schema abstraction" in a multiple-trace memory model. Psychological Review, 93, 411-458.

KruschKe, J. K. (1992). Alcove: An exemplar-based connectionist model of category learning. Psychological Review, 99, 22-44.

KruschKe, J. K. (1996). Dimensional relevance shifts in category learning. Connection Science, 8, 225-247.

Lafond, D., Lacouture, Y., \& Mineau, G. (2007). Complexity minimization in rule-based category learning: Revising the catalog 
of Boolean concepts and evidence for nonminimal rules. Journal of Mathematical Psychology, 51, 57-74.

Love, B. C., Medin, D. L., \& Gureckis, T. M. (2004). Sustain: A network model of category learning. Psychological Review, 111, 309332.

Medin, D. L., \& Bettger, J. G. (1994). Presentation order and recognition of categorically related examples. Psychonomic Bulletin \& Review, 1, 250-254

Medin, D. L., \& Schaffer, M. (1978). A context theory of classification learning. Psychological Review, 85, 207-238.

Nosofsky, R. M., Gluck, M. A., Palmeri, T. J., McKinley, S. C., \& Glauthier, P. (1994). Comparing models of rules-based classification learning: A replication and extension of Shepard, Hovland, and Jenkins (1961). Memory \& Cognition, 22, 352-369.

Nosofsky, R. M., Palmeri, T. J., \& McKinley, S. C. (1994). Ruleplus-exception model of classification learning. Psychological Review, 101, 53-79.

RosseEL, Y. (2002). Mixture models of categorization. Journal of Mathematical Psychology, 46, 178-210.

SaKamoto, Y., Jones, M., \& Love, B. C. (2008). Putting the psychology back into psychological models: Mechanistic versus rational approaches. Memory \& Cognition, 36, 1057-1065.

SaKamoto, Y., \& Love, B. C. (2004). Schematic influences on category learning and recognition memory. Journal of Experimental Psychology: General, 133, 534-553.

Shepard, R. N., Hovland, C. L., \& Jenkins, H. M. (1961). Learning and memorization of classifications. Psychological Monographs, 75, 13, Whole No. 517.

Smith, E. E., \& Sloman, S. A. (1994). Similarity-versus rule-based categorization. Memory \& Cognition, 22, 377-386.

Stewart, N., Brown, G. D. A., \& Chater, N. (2002). Sequence effects in categorization of simple perceptual stimuli. Journal of Experimental Psychology: Learning, Memory, \& Cognition, 28, 3-11.

VIGO, R. (2006). A note on the complexity of Boolean concepts. Journal of Mathematical Psychology, 50, 501-510.

\section{NOTES}

1. Many studies require only $75 \%$ or $80 \%$, but such a low criterion would defeat the goals of our study, because it would make it possible to learn only the "rule-like" examples and completely avoid the "exceptions."

2. In four Boolean dimensions, there are 74 qualitatively different types of concepts with 8 positive examples, giving a classification analogous to (although more complex than) that in Shepard et al. (1961).

3. The term rule-based refers to the fact that this order respects a rule plus exception organization (Nosofsky, Palmeri, \& McKinley, 1994). The organization more generally relates to disjunctive normal form, in which each term indicates a conjunction of features. Some disjunctive terms cover many cases (major rules); others cover fewer (minor rules, or major classes of exceptions); and still others cover only one case each (exceptions). An example is a rule like "birds $=$ (animals that fly) or (ostrich-like animals - which possess feathers but do not fly) or (kiwialso a flightless bird but not like an ostrich)."

4. Note that similarity is computed on a trial-by-trial basis, so although interitem similarity is always maximal between successive examples, it is not necessarily maximized over an entire block (i.e., similarity is maximized locally but not globally).

5. The average interitem similarity was computed for positive examples only because negative examples were presented in random order in all presentation orders.

6. The loss of subjects probably stems from our subject-scheduling system, which unfortunately did not leave many subjects sufficient time to complete the experiment, given the strict $100 \%$ criterion. In their second study, Elio and Anderson (1984) also excluded 14 cases among 80 subjects with an $85 \%$ correct criterion.

(Manuscript received March 24, 2009; revision accepted for publication June 25, 2009.) 